\title{
Emerging Contaminants - Small Concentrations, Big Problems
}

\section{Satinder K. Brar}

\section{Assistant Professor, INRS-ETE, Université du Québec Centre Eau, Terre \& Environnement/Centre for Water, Québec, Canada}

For the last decade, we have seen an upsurge in the scientific documentation of emerging contaminants which comprises several chemical and microbial constituents that have not traditionally been considered as contaminants and are present in the environment on a global scale. Emerging contaminants can originate from a variety of animal- and human-waste sources, including municipal, agricultural, and industrial wastewater pathways. The emerging contaminants comprise, nanoparticles, endocrine disrupter compounds, pharmaceuticals and personal care products, prions, cyanobacteria, plasticizers, organometallic compounds, among others. The trail to the environment can be from rapid and direct discharges of effluent into a water body or the slow leaching of stored waste through soils. These emerging contaminants represent a shift in traditional thinking, as many are produced industrially yet are dispersed to the environment from domestic, commercial, and industrial uses. The emerging contaminants present the biggest challenge of analysis as some of them are present in nanograms or pictograms and in fact, these environmentally relevant concentrations lend toxicity.

The emerging contaminants once on their pathway into the wastewater treatment plants encounter various other contaminants of different forms, such as organic, inorganic and microorganisms. Let's discuss the trail of the emerging contaminant into a typical wastewater treatment plant. It commences with the primary treatment process which comprises physical-chemical treatment where the emerging contaminants can undergo simple partitioning between the solid and liquid portions based on their octanol-water coefficient values. This can lead to removal of the contaminants from the unit operations causing re-distribution in the primary effluent and primary sludge rather than treatment. Likewise, the effluent entering the secondary treatment process can undergo biological degradation again based on toxicity, solubility and biodegradability. Secondary treatment can also result in formation of secondary by-products from the precursor emerging contaminant which can present enhanced toxicity problems as compared to the initial compound. Finally, the untransformed contaminant will also pass through the sludge treatment steps, comprising dewatering, incineration and land application. The contaminant left in the treated secondary effluent will be further significantly transformed during tertiary treatment steps of disinfection. The enigma of emerging contaminant fate does not end here; it undergoes further disposal or reutilization of wastewater sludge leading to diverse contamination of various environmental media, ranging from soil to water.

While undergoing various treatment steps, the emerging contaminants are in close interaction with other organic as well as inorganic contaminants. These interactions can lead to either synergistic or antagonistic effect, in other words, either they can enhance the degradation of the emerging contaminant or inhibit the degradation rate. The trend is very important in the sense that it can determine the ultimate fate of the emerging contaminants. When talking about inorganic contaminants, most evident ones are heavy metals. There is possibility of complexation and many other interactions which can now determine the ultimate fate of the modified emerging contaminants. Thus, the equation of emerging contaminant interaction with other entities present in the wastewater is not so simple and the result can be quite intriguing. Likewise, the physical state of the wastewater and wastewater sludge which the science translates in terms of viscosity, particle size can also affect their distribution from one phase into another either enhancing their degradation reaction kinetics or completely subduing it ! For example, as the emerging contaminant traverses from primary to secondary treatment, there is a drop in viscosity which favors many reactions further ameliorating its removal or modification (into other complex products by virtue of its reaction with other contaminants) rate. Further, the transport of these unmodified or modified emerging contaminants in the living organisms is another enigma due to lower concentrations, yet significant reactions!

Thus, future course of these emerging contaminants in our environment, such as wastewater and further downstream into rivers, soils, among others need to be very closely studied through a multidisciplinary approach. The multi-disciplinary team can comprise, chemists, biologists, physicists, physiologists, environmentalists, medical specialists, among many others. In fact, in order to leave this planet safe and sane for the future generation, we have a responsibility to reckon by minimizing the so called plastic life and enhancing nature oriented products, in turn propagating a clean and green environment.

*Corresponding author: Satinder K. Brar, Assistant Professor, INRS-ETE, Université du Québec Centre Eau, Terre \& Environnement/Centre for Water, Québec, Canada, E-mail: satinder.brar@ete.inrs.ca

Received June 27, 2011; Accepted July 01, 2011; Published July 15, 2011

Citation: Brar SK (2011) Emerging Contaminants - Small Concentrations, Big Problems. Hydrol Current Res 2:101e. doi:10.4172/2157-7587.1000101e

Copyright: (c) 2011 Brar SK. This is an open-access article distributed under the terms of the Creative Commons Attribution License, which permits unrestricted use, distribution, and reproduction in any medium, provided the original author and source are credited. 